\title{
Editorial
}

\section{Innovations and challenges across the heart failure spectrum}

\author{
Paulino Alvarez ${ }^{1,2}$, Alexandros Briasoulis ${ }^{3,4, *}$ \\ ${ }^{1}$ Department of Cardiovascular Medicine, Heart, Vascular, and Thoracic Institute, Cleveland Clinic, Cleveland, OH 44195, USA \\ ${ }^{2}$ Division of Heart failure and Cardiac Transplantation, Cleveland Clinic, Cleveland, OH 44195, USA \\ ${ }^{3}$ Division of Heart Failure and Transplant, University of Iowa Hospitals and Clinics, Iowa, IA 52242, USA \\ ${ }^{4}$ Department of Clinical Therapeutics, National and Kapodistrian University, 11528 Athens, Greece \\ *Correspondence: alexbriasoulis@gmail.com; ALVAREP3@ccf.org (Alexandros Briasoulis) \\ Academic Editor: Peter A. McCullough \\ Submitted: 4 December 2021 Accepted: 7 December 2021 Published: 19 January 2022
}

Keywords: Heart failure; Outcomes; Pulmonary hypertension

Heart failure is a global problem that affects 38 million patients around the world [1]. Regardless of the etiology, patients with heart failure experience limitation of their functional capacity, symptoms related to congestion and/or low output, a decrease in quality of life and a reduction in their life expectancy [2]. Given that ischemic heart disease and hypertensive heart disease are the most common causes of heart failure prevention and treatment of those conditions are essential to reduce its incidence [3]. Once structural heart abnormalities and symptoms develop prevention of congestion, initiation of guideline directed medical therapy, use of devices and timely referral for advanced heart failure therapies become the focus to improve clinical outcomes [4].

The trajectories of heart failure patients are heterogeneous. Because of that personalized follow up is necessary [5]. The COVID-19 pandemic changed the landscape of medicine forever. Social distancing and the fear of turning health care facilities as virus transmission hubs motivated a drastic reduction in in-person encounters [6]. In this context telemedicine has evolved as a necessary tool in everyday practice and chronic disease management systems in heart failure are an excellent platform for its implementation. In this issue we discuss principles of application of tele monitoring to maximize patient trajectory tracking and minimize in person visits [7].

Advances in the last decade has defined the four pillars of pharmacological therapy in heart failure with reduced ejection fraction [4]. The implementation of quadruple therapy (Heart failure specific beta-blocker + ARNI or ARB or ACEI) + Aldosterone Blocker + SGLT-2 inhibitor) has shown a consistent improvement in survival [8]. However elderly patients and those with advanced renal disease have been unrepresented from pivotal clinical trials. Kolben et al. [9] will discuss the considerations in the implementation of those therapies specific patient population.

Pulmonary hypertension is associated with increased mortality in heart failure. No specific therapies for Group 2 pulmonary hypertension have been approved [10]. Functional mitral regurgitation is associated with increased mor- tality in patients with heart failure [11]. Functional mitral regurgitation is a disease of the left ventricle and GDMT is the first step in treatment [12]. Percutaneous repair of the mitral valve has a Class $2 \mathrm{~A}, \mathrm{LOE} \mathrm{B}$ recommendation for patients with heart failure, LVEF $<50 \%$ with severe chronic secondary MR and persistent severe symptoms in spite of optimal medical therapy [13]. Mandurino-Mirizzi et al. [14] will tackle the complex interaction of percutaneous edge to edge repair, mitral regurgitation, pulmonary hypertension and possible future directions.

Atrial fibrillation is the most common type of arrhythmia in heart failure, is associated with worse prognosis [15]. Since the introduction of pulmonary vein isolation by Haisaguirre et al. [16] the procedure and technical advances have evolved and the center of gravity has been moving from medical therapy and rate control to interventional therapy and rhythm control. This approach has translated not only in nicer EKGs but in improvements in clinical outcomes with reductions in hospitalizations and mortality [17]. Cardiovascular implantable electronic device (CIED) such as implantable cardioverter defibrillators (ICDs) and cardiac resynchronization therapy (CRT) are part of the armamentarium to decrease mortality in heart failure. However those devices are not free from complications and advances in patient selection to improve the risk/benefit ratio of the device selection is necessary. Sohrabi et al. [18] will guide us in the current implementation of electrophysiological therapies in our heart failure patients.

In patients with refractory symptoms advanced heart failure therapies and palliative care should be considered. Tatum et al. [19] describe the amazing evolution of durable mechanical support that motivated changes in the heart allocation policy and the consequences of those changes in the future of durable mechanical support.

Our hope is that this issue Reviews in Cardiovascular Medicine will motivate new questions and applications for the benefit of our patients. 


\section{Author contributions}

Authors equally contributed to the content.

\section{Ethics approval and consent to participate}

Not applicable.

\section{Acknowledgment}

Not applicable.

\section{Funding}

This research received no external funding.

\section{Conflict of interest}

The authors declare no conflict of interest.

\section{Disclosures}

No relevant disclosures.

\section{References}

[1] Braunwald E. The war against heart failure: the Lancet lecture. Lancet. 2015; 385: 812-824.

[2] Thibodeau JT, Drazner MH. The Role of the Clinical Examination in Patients with Heart Failure. JACC: Heart Failure. 2018; 6: $543-551$

[3] Bragazzi NL, Zhong W, Shu J, Abu Much A, Lotan D, Grupper A, et al. Burden of heart failure and underlying causes in 195 countries and territories from 1990 to 2017. European Journal of Preventive Cardiology. 2021; zwaal47.

[4] McDonagh TA, Metra M, Adamo M, Gardner RS, Baumbach A, Böhm M, et al. 2021 ESC Guidelines for the diagnosis and treatment of acute and chronic heart failure: Developed by the Task Force for the diagnosis and treatment of acute and chronic heart failure of the European Society of Cardiology (ESC) With the special contribution of the Heart Failure Association (HFA) of the ESC. European Heart Journal. 2021. 42: 3599-3726.

[5] Desai AS, Stevenson LW. Rehospitalization for Heart Failure. Circulation. 2012; 126: 501-506.

[6] Werner RM, Glied SA. Covid-Induced Changes in Health Care Delivery - can they last? New England Journal of Medicine. 2021; 385: 868-870.

[7] Alvarez P, Sianis A, Brown J, Ali A, Briasoulis A. Chronic disease management in heart failure: focus on telemedicine and remote monitoring. Reviews in Cardiovascular Medicine. 2021; 22: 403-413.

[8] Vaduganathan M, Claggett BL, Jhund PS, Cunningham JW, Pedro Ferreira J, Zannad F, et al. Estimating lifetime benefits of comprehensive disease-modifying pharmacological therapies in patients with heart failure with reduced ejection fraction: a comparative analysis of three randomised controlled trials. The Lancet. 2020; 396: 121-128.

[9] Kolben Y, Kessler A, Puris G, Nachman D, Alvarez P, Briasoulis A, et al. Management of heart failure with reduced ejection fraction: challenges in patients with atrial fibrillation, renal disease and in the elderly. Reviews in Cardiovascular Medicine. 2022; 23: 016

[10] Vachiéry J, Tedford RJ, Rosenkranz S, Palazzini M, Lang I, Guazzi M, et al. Pulmonary hypertension due to left heart disease. European Respiratory Journal. 2019; 53: 1801897.

[11] Bartko PE, Heitzinger G, Pavo N, Heitzinger M, Spinka G, Prausmüller S, et al. Burden, treatment use, and outcome of secondary mitral regurgitation across the spectrum of heart failure: observational cohort study. BMJ-British Medical Journal. 2021; 373: n1421.

[12] Goliasch G, Bartko PE, Pavo N, Neuhold S, Wurm R, Mascherbauer J, et al. Refining the prognostic impact of functional mitral regurgitation in chronic heart failure. European Heart Journal. 2018; 39: 39-46.

[13] Otto CM, Nishimura RA, Bonow RO, Carabello BA, Erwin JP 3rd, Gentile F, et al. 2020 ACC/AHA Guideline for the Management of Patients With Valvular Heart Disease: A Report of the American College of Cardiology/American Heart Association Joint Committee on Clinical Practice Guidelines. Circulation. 2021; 143: e72-e227.

[14] Mandurino-Mirizzi A, Tua L, Arzuffi L, Demarchi A, Somaschini A, Tournas G, et al. Transcatheter mitral valve repair with MitraClip in patients with pulmonary hypertension: hemodynamic and prognostic perspectives. Reviews in Cardiovascular Medicine. 2021; 22: 33-38.

[15] Anter E, Jessup M, Callans DJ. Atrial Fibrillation and Heart Failure. Circulation. 2009; 119: 2516-2525.

[16] Haïssaguerre M, Jaïs P, Shah DC, Takahashi A, Hocini M, Quiniou $\mathrm{G}$, et al. Spontaneous initiation of atrial fibrillation by ectopic beats originating in the pulmonary veins. The New England Journal of Medicine. 1998; 339: 659-666.

[17] Marrouche NF, Brachmann J, Andresen D, Siebels J, Boersma L, Jordaens L, et al. Catheter Ablation for Atrial Fibrillation with Heart Failure. New England Journal of Medicine. 2018; 378: 417-427.

[18] Sohrabi C, Ahsan S, Briasoulis A, Androulakis E, Siasos G, Srinivasan NT, et al. Contemporary management of heart failure patients with reduced ejection fraction: the role of implantable devices and catheter ablation. Reviews in Cardiovascular Medicine. 2021; 22: 415-428.

[19] Tatum RT, Massey HT, Tchantchaleishvili V. Impact of mechanical circulatory support on donor heart allocation: past, present, and future. Reviews in Cardiovascular Medicine. 2021; 22: 2532 . 